Check for updates

Cite this: RSC Adv., 2017, 7, 35160

\title{
Surfactant-driven direct synthesis of a hierarchical hollow MgO nanofiber-nanoparticle composite by electrospinning $\dagger$
}

\author{
Shijina S. Sainudeen, Lakshmi B. Asok, Anitta Varghese, A. Sreekumaran Nair \\ and Gopi Krishnan (D) *
}

\begin{abstract}
A hierarchical structure comprising decorated faceted nanoparticles (NPs) on hollow nanofibers (NFs) combines the advantages of large surface areas of NPs and anisotropic properties of hollow NFs. Such particular combinations are desirable for applications in batteries, dye-sensitized solar cells (DSSCs) and catalysis. Herein we report a facile surfactant-driven unprecedented fabrication of hollow $\mathrm{MgO}$ nanofibers (NFs) decorated with faceted $\mathrm{MgO}$ nanoparticles (NPs) via an electrospinning-thermal treatment process. The MgO NF/NP composite was achieved in one step by adding a cationic surfactant, cetyl trimethyl ammonium bromide (CTAB), to a solution containing a polymer and an MgO precursor followed by electrospinning and calcination of the electrospun fibers in air. Tuning of the MgO NP size and faceting of the NPs on the fiber surface was achieved by increasing the concentration of CTAB in the solution used for electrospinning. In particular, increasing concentration and order of CTAB addition to the spinning solution influences the MgO NPs decoration and coverage on the nanofibers, indicating the possibility that a well-defined choice of NP density can be achieved. The faceted particles show cube-, octahedron- and rod-like morphology depending on the growth rate of the $\langle 100\rangle$ and $\langle 111\rangle$ directions. Furthermore, by employing a mixed solvent (methanol + DMF) strategy, we were able to reduce the size distribution and control the shape of the faceted NPs only to octahedra. Moreover a tentative mechanism for the formation of hollow MgO NFs and faceted NPs using TGA and FTIR analysis has also been proposed. This new approach of surfactant driven electrospinning of a NP decorated NF composite will have a strong impact on various applications as it is simple, effective and straightforward.
\end{abstract}

Received 23rd May 2017

Accepted 7th July 2017

DOI: 10.1039/c7ra05812h

rsc.li/rsc-advances important applications, e.g. in catalysis, where the shape and size of the nanoparticles has a profound influence in the catalytic reactivity. ${ }^{6}$ Moreover, reactivity of the NPs can also be enhanced by faceting, such as the increased reactivity of metal (Pt, $\mathrm{Pd}$ and $\mathrm{Au})$ and metal oxide $\left(\mathrm{Cu}_{2} \mathrm{O}, \mathrm{Fe}_{2} \mathrm{O}_{3}, \mathrm{Co}_{3} \mathrm{O}_{4}, \mathrm{TiO}_{2}\right.$, $\mathrm{SnO}_{2}$ ) NPs. ${ }^{7-14}$

One-dimensional (1D) nanostructures such as nanofibers and nanorods possess' unique density of states that leads to different optical, electronic and magnetic properties compared to other dimensional nanostructures and their bulk counterparts. Therefore they have high demand in various applications like water treatment, energy, catalysis and gas sensing. ${ }^{15-23}$ They are also attractive due to their enhanced electron and phonon transport properties, inherent to their unique dimension. Though various techniques are available for the synthesis of nanofibers, electrospinning is considered as a versatile technique for its capability in large-scale synthesis of polymer and ceramic fibers. ${ }^{24-27}$ Recently, the advantages of combining both 0D (nanoparticles) and 1D (nanofiber/nanowire) nanostructures have been studied for various applications, such as, lithium ion batteries, DSSC and catalysis..$^{\mathbf{6 1 5 - 2 3 , 2 8}}$ Synthesis of hierarchical 
structures have been reported by combining electrospinning and hydrothermal, ${ }^{29}$ co-electrospinning-electrospraying, ${ }^{30}$ modified template-assisted method ${ }^{31}$ and also by step-wise hydrothermal approach. ${ }^{32}$ Li et al. proposed the synthesis of hybrid core-branch nanoarchitecture by integrating $\mathrm{Fe}_{2} \mathrm{O}_{3}$ nanoneedles on ultrafine Ni nanotube arrays (NiNTAs). ${ }^{31}$ The synthesis technique was based on a modified template-assisted method, which employed a bottom-up strategy starting from ultrafine $\mathrm{ZnO}$ nanorod arrays. These nanorod arrays ensured the formation of ultrafine $\mathrm{Ni}$ nanotube arrays with ultrathin tube walls. Thus developed NiNTAs@ $@ \mathrm{Fe}_{2} \mathrm{O}_{3}$ nanoneedle electrode demonstrated to be a highly capacitive anode for asymmetric supercapacitors. Xia et al. also reported a solution-based synthesis of hierarchical heterostructures of $\mathrm{Ag}$ nanoparticles decorated $\mathrm{MnO}_{2}$ nanowires as promising electrodes for supercapacitors. $^{28}$ A stepwise hydrothermal approach for the synthesis of hierarchical $\mathrm{TiO}_{2}-\mathrm{B}$ nanowire@ $\alpha-\mathrm{Fe}_{2} \mathrm{O}_{3}$ nanothorn core-branch arrays with improved performance as a high capacity anode for lithium-ion batteries was reported by Xia et $a .^{32}$ In addition, colloidal-electrospinning has also been employed where organic/inorganic nanoparticles are dispersed in a polymer-precursor solution and electrospun. ${ }^{16,30-39}$ For instance Formo et $a l .{ }^{33}$ have reported the functionalization of electrospun $\mathrm{TiO}_{2}$ nanofibers with $\mathrm{Pt}$ nanoparticles and nanowires by this method. They showed an increased catalytic activity for the hydrogenation of azo bonds in methyl red. Anjusree et al. has reported the synthesis of $\mathrm{TiO}_{2}$ nanofiber-TiO ${ }_{2}$ nanoparticle composite via combination of electrospraying and electrospinning for dye-sensitized solar cell (DSSC) applications. ${ }^{30}$ The efficiency of the solar cell is substantially enhanced by the hierarchical structure. Nam et al. reported the synthesis of $\mathrm{Ag} / \mathrm{Au}$ nanoparticle-embedded $\mathrm{TiO}_{2}$ nanofiber composite via electrospinning for use in lithium ion batteries. ${ }^{39}$ Moreover, a simple synthesis methodology for such combinatory process with good control of NP can be more beneficial for scaling-up the procedure for various practical applications.

In this paper, the concept and value of hierarchical structures is further improved by considering a single-step processing of hierarchical structures of nanoparticles with hollow fibers/nanotubes. Hollow nanostructures offer advantages in terms of electronic activity and surface area enhancements with the possibility of incorporating the nanoparticles both inside and external to the walls. Further, for the first time, the shape, faceting and decoration of NP on NFs are controlled by the unprecedented use of suitable surfactants that can enhance the catalytic activity of the particles. Moreover, the added advantage of faceted nanoparticles in combination with 1D can offer new multi-functionalities for applications such as catalysis, batteries, DSSC and gas sensing. ${ }^{6,15-23,30-39}$ We report for the first time a direct synthesis of hollow MgO NFs decorated with faceted MgO NPs via electrospinning, where we combine both these dimensions in a single nanoarchitecture just by the addition of a surfactant. Since $\mathrm{MgO}$ is an important material, which finds application in catalysis, water treatment, refractory material, photocatalysis and as an adsorbent in separation of $\mathrm{CO}_{2}$ from the flue gases, ${ }^{40-45}$ we have chosen $\mathrm{MgO}$ as the model system for our interest.

\section{Experimental}

\section{Synthesis of nanofibers}

The viscous solution for electrospinning was prepared by first adding $20 \mathrm{wt} \%$ of PVP to $10 \mathrm{~mL}$ of solvent (DMF) and stirred for $15 \mathrm{~min}$ to obtain a uniform clear solution. Later $16 \mathrm{wt} \%$ of precursor $\left(\mathrm{Mg}(\mathrm{Ac})_{2} \cdot 4 \mathrm{H}_{2} \mathrm{O}\right)$ was added to the clear polymer solution while stirring. About $0.75 \mathrm{~g}$ CTAB was added to the above solution mixture and stirred for $5-15 \mathrm{~h}$ to increase the stability of solution. After stirring, the final solution obtained was turbid as the solubility of CTAB is less in DMF and for making it a completely clear solution, it was further stirred at $35{ }^{\circ} \mathrm{C}$ for $5-10$ minutes before electrospinning. The viscous precursor solution was taken in a $20 \mathrm{~mL}$ syringe with $0.90 \times 38$ mm needle attached to it. A $29 \mathrm{kV}(+25 \mathrm{kV}$ and $-4 \mathrm{kV})$ voltage was applied between the needle tip and the aluminium foil collector, which was maintained at a distance of $17 \mathrm{~cm}$. Humidity and temperature inside the electrospinning chamber were maintained at $20 \%$ and $25{ }^{\circ} \mathrm{C}$, respectively. The solution was pumped into the needle tip inside the chamber at a flow rate of $1 \mathrm{~mL} \mathrm{~h}^{-1}$. The as-prepared $\mathrm{Mg}(\mathrm{Ac})_{2} \cdot 4 \mathrm{H}_{2} \mathrm{O} / \mathrm{PVP} / \mathrm{CTAB}$ composite fiber was then sintered in air at $500{ }^{\circ} \mathrm{C}$ for $12 \mathrm{~h}$ to obtain hollow $\mathrm{MgO}$ nanofibers with faceted $\mathrm{MgO}$ nanoparticles on the fiber surface.

\section{TEM analysis}

TEM analysis of MgO NFs/NP composite fiber was done using FEI Tecnai - F20 operating at $200 \mathrm{kV}$. TEM sample was prepared by placing a few pieces of MgO NFs/NP powder on carboncoated copper grid and by gently pressing such that they adhere strongly to the surface.

\section{Results and discussion}

Fig. 1 shows bright-field transmission electron microscopy (TEM) analysis of hollow MgO nanofibers synthesized via electrospinning without the addition of CTAB. The MgO fibers were clearly identified of being hollow from the contrast difference arising in the image, which is uniform for all the fibers. Selected-area electron diffraction (SAED) analysis indeed confirms the presence of $\mathrm{MgO}$ fibers with face centered cubic structure. It also confirms the polycrystalline nature of the fibers. The interplanar distance ' $d$ ' obtained from the diffraction pattern are $2.43 \AA$ for $\left(\begin{array}{lll}1 & 1 & 1\end{array}\right), 2.1 \AA$ for $\left(\begin{array}{lll}2 & 0 & 0\end{array}\right)$ and $1.48 \AA$ for ( $\left.\begin{array}{lll}2 & 2 & 0\end{array}\right)$ planes, respectively, which matches exactly with the JCPDS data of MgO (reference no. 78-0430). TEM analysis shows that almost all the fibers prepared are hollow after calcination in air medium compared to previous reported work on $\mathrm{MgO}$ showing the combination of hollow and solid fibers. ${ }^{46}$

Fig. 2(a)-(c) show the bright-field TEM images of the MgO nanofibers (electrospun with the CTAB surfactant) calcined at $500{ }^{\circ} \mathrm{C}$. In comparison with Fig. 1, TEM image of MgO NFs (spun with CTAB) show the presence of NPs on the surface of MgO NFs. This clearly indicates surface modification of the NFs with the addition of the surfactant (CTAB). The fiber diameter is reduced in comparison to the fibers produced without the 


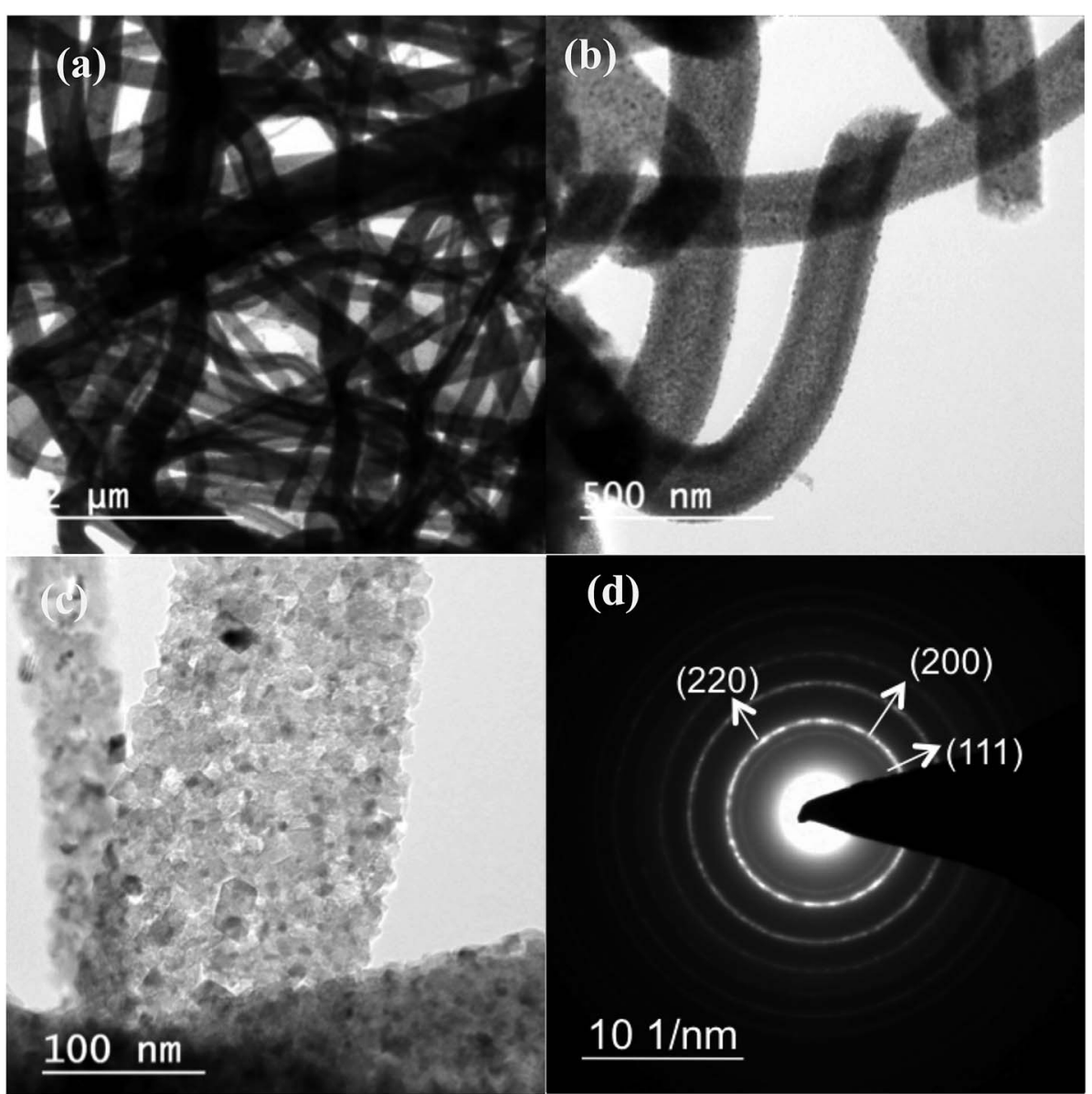

Fig. 1 (a), (b) $\&$ (c) shows the bright-field TEM image of hollow MgO NFs at different magnification synthesized without addition of CTAB. (d) Selected Area Electron Diffraction (SAED) pattern of the MgO nanofibers.

addition of CTAB. Detailed investigation of the fiber surface was also done using field-emission scanning electron microscopy (FESEM) (Fig. 2(e) and (f) \& 3(c) and (f)). FE-SEM images confirm the presence of faceted nanoparticles decorated on the fiber surface. TEM images also clearly show that the particles that modify the surface are faceted. The faceted particles showed various projections on the fiber surface with non-uniform growth of the particles. They also appeared in various shapes like cube, octahedron, rod, etc. In addition, the TEM images confirm that the fibers are hollow and the diameter of the fibers varied from 200-400 $\mathrm{nm}$. Selected area electron diffraction pattern of the sintered fiber (shown in Fig. 2(a) inset) confirms that the fiber is indeed MgO. The high-resolution electron microscopy (HRTEM) image in Fig. 2(d) shows the MgO (111) plane with an interplanar distance of $0.245 \mathrm{~nm}$, which matches with pure MgO. Energy dispersive X-ray analysis (EDX) of the faceted nanoparticles also confirms that the faceted NPs are indeed MgO (ESI Fig. S3†).

The decrease in fiber diameter with the addition of CTAB could be due to the increased charge density of the solution that increases the motion instability, leading to a longer spiral path of the drawn fibers to reach the target. ${ }^{47}$ The nanofiber diameter usually decreases with increase in the surfactant concentration to the electrospinning solution. ${ }^{48,49}$ This is due to the fact that the surfactant forms micelles in the solution thereby forming polymer-surfactant complex. The complex thus formed has a major role in the solution properties, which in turn affects the fiber morphology. It increases the solution viscosity and conductivity but decreases the surface tension. Our experiment also confirms that increasing the concentration of CTAB from $0.1 \mathrm{~g}$ to $0.75 \mathrm{~g}$ shows the reduction of fiber diameter from $\sim 400 \mathrm{~nm}$ to $\sim 200 \mathrm{~nm}$ (ESI Fig. S4 $\dagger$ ) which is in contradiction with the viscosity readings of the solution that shows an increasing trend (ESI Table $1 \dagger$ ). Normally the diameter of the fibers increases with the increase in viscosity of the spinning solution. ${ }^{26}$ It is evident that size distribution and faceting of the NPs were better controlled with increasing CTAB concentration. Moreover, at $0.75 \mathrm{~g}$ addition of CTAB, NP size reduction and a better size distribution of NPs on NF surface have been observed. Furthermore, the faceting of nanoparticles also tends to be distinct and highly pronounced with increasing concentration of CTAB for e.g. $0.75 \mathrm{~g}$ (ESI Fig. S5 $\dagger$ ). The above analysis was based on our experimental results that confirm the faceting of nanoparticles only after $0.25 \mathrm{~g}$ of the CTAB addition to the solution as the $0.1 \mathrm{~g}$ shows only spherical particles. This is due to increased concentration of CTAB molecules that increases the micellization process and number of micelles formed, which has a pronounced effect on the growth of particles as they 

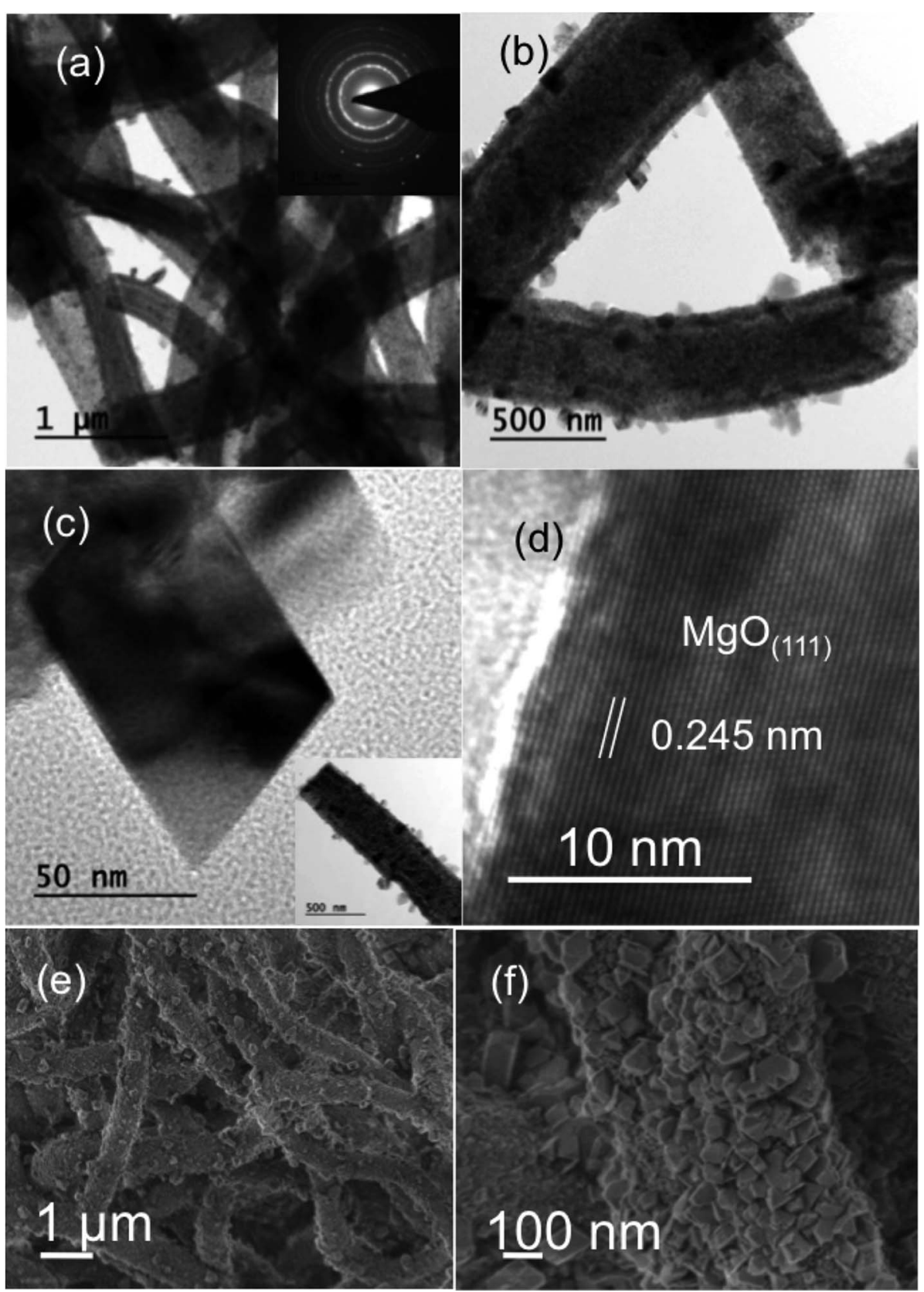

Fig. 2 (a) \& (b) shows the bright-field TEM image of hollow MgO NFs/NPs composite fiber at different magnification synthesized with addition of CTAB. Inset in (a) shows the diffraction pattern of the MgO NFs/NPs composite, which matches with interplanar distance "d" of MgO. (c) High magnification bright-field TEM image of the faceted MgO NPs on the fiber surface. Inset shows a single fiber TEM image, of which the faceted MgO NPs is shown in figure. (d) High resolution TEM image of the MgO NPs formed on the surface of fiber. (e, f) shows the SEM images of the $\mathrm{MgO}$ NF/NP composite.

tend to be adsorbed on them. Now the capping effect of CTAB also increases with the increase in the concentration thereby making the particles faceted..$^{50}$ Moreover, increased number of micelles in the solution also leads to a decrease in particle size as well as observed with increase in CTAB addition.

In order to study the effect of surfactant (CTAB) on the formation of faceted particles and the subsequent surface modification, we have changed the order of CTAB addition to the electrospinning solution. First CTAB- $\mathrm{Mg}(\mathrm{Ac})_{2} \cdot 4 \mathrm{H}_{2} \mathrm{O}$ were added followed by PVP to make the electrospinning solution and the corresponding calcined NFs were analyzed using brightfield TEM imaging. Results shown in Fig. 3(a)-(c) confirm that, when the reagents were added in the above-mentioned order, more particles were observed on the fiber surface and the faceting was also uniform, as most of them appeared cube and rod in shape. Fig. 3(b) and (c) shows that the NP decorated on the surface appears to cover around the NFs similar to a core/shell structure of $\mathrm{MgO}$ fiber core/MgO NP shell. In addition, the coverage of the NP on the fiber is more dense and uniform. The observed structure and phenomenon could be due to increased 

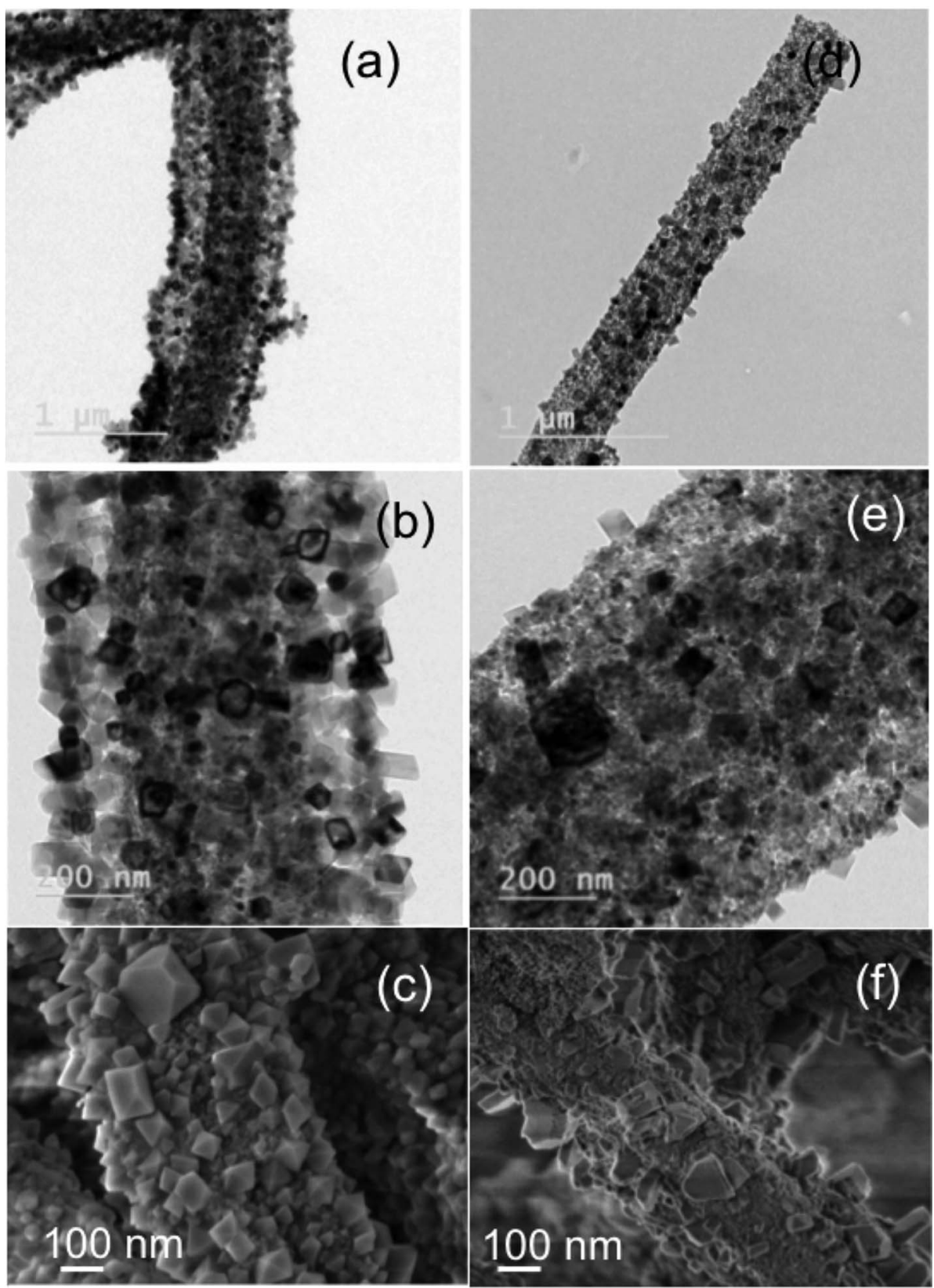

Fig. 3 Bright-field TEM and SEM images of calcinated fibers with different order of CTAB addition. (a, b, c) shows NF/NP composite formation with addition of CTAB and metal precursor prior to polymer. (d, e, f) shows the effect of NF/NP formation with metal precursor and polymer addition prior to CTAB.

interaction of precursor molecules $\left(\mathrm{Mg}(\mathrm{Ac})_{2} \cdot 4 \mathrm{H}_{2} \mathrm{O}\right)$ with $\mathrm{CTAB}$ (resulting in complete micelle formation) than with the polymer resulting from the order of addition. Fig. 3(d)-(f) shows that when the order is changed/reversed with precursor added first followed by polymer and then CTAB, the conformal and dense coating disappeared and only a surface modification by faceted NPs is observed. From the above-mentioned observation, it is evident that the order of CTAB addition has an influence on the NPs distribution and decoration on NFs.

Critical nuclei that act as a seed for NP formation are generally polyhedral in shape in order to minimize the surface energy. Similarly the critical nucleus of an FCC for NP nucleation is a truncated cuboctahedron as it has a minimum energy barrier to form a critical nucleus. This is an equilibrium shape, which contribute to the formation of cube and octahedron shapes that can be observed from Fig. 2. Moreover, these shapes, which can be realized by kinetically controlling the relative growth rates of facets, are completely dependent on the preferential adsorption of CTAB on $\{100\}$ or $\{111\}$ facets. The ratio of growth rate along $\langle 100\rangle$ and $\langle 111\rangle$ direction of the seed crystal will determine the final shape of particles as cube, octahedron and rod as shown in the TEM images of Fig. 2 and 3. The $\{100\}$ facets enclose cube shape as a consequence of preferred growth along $\langle 111\rangle,\{111\}$ facets enclose octahedral as 
a preferential growth in $\langle 100\rangle$, rod shapes are observed due to uniaxial continuous growth in [111]. Furthermore from Fig. 3, formation of uniform faceted NPs in shape with different projection on the fiber surface could also be observed.

Now to see the effect of solvent's role in surface modification, we have prepared the precursor/PVP/CTAB electrospinning solution not only in DMF but also in ethanol. Results with ethanol also showed the surface modification and NPs decoration on NF morphology similar to that of DMF (ESI Fig. S6 $\dagger$ ). Moreover, faceting of the particles and their distribution was not as similar to the observation with pure DMF solvent.

Further by employing a solvent mixture strategy of (DMF + methanol) in $50: 50$ ratio instead of pure DMF solvent, the NP decorated NF morphology is not only preserved but at the same time the size of the MgO NP decorated on the fiber is decreased to $\sim 40$ to $60 \mathrm{~nm}$ as compared to $\sim 80$ to $90 \mathrm{~nm}$ with pure DMF. More importantly the faceting and shape of the NPs as shown in Fig. 4(a) and (b) are very well controlled to only octahedral shape, which was difficult to achieve in the case of pure DMF. The NPs are enclosed with $\{111\}$ facets. We have chosen methanol as one of the solvents in combination to DMF as CTAB is completely soluble in methanol. Here methanol is more polar than DMF and it belongs to polar protic solvent category compared to polar aprotic nature of DMF, which increases the overall polarity of the solution hence enhances and controls the micelle formation. ${ }^{51-53}$ Please note that the heating of spinning solutions to $35{ }^{\circ} \mathrm{C}$ prior to electrospinning was mandatory to achieve the faceted particles on the fiber surface in all the cases shown in Fig. 2-4. In addition we have tried also with a solvent mixture of (DMF + ethanol) in a 50:50 ratio but the control of particle size and shape that was achieved with (methanol + DMF) were not observed in the former case.

Now, in order to understand a plausible mechanism, we will try to obtain more insight in to the NP/NF formation. In general, addition of CTAB to the electrospinning solution forms micelles below or near to CMC (Critical Micelle Concentration) and also forms PVP-CTAB complex in DMF. ${ }^{53,54}$ Moreover, PVP also bonds with $\mathrm{Mg}^{2+}$ ions from the metal precursor and leads to the formation of hollow fiber morphology as explained below, whereas $\mathrm{CTA}^{+}$- head group of CTAB bonds with the hydroxyl group of $\mathrm{Mg}(\mathrm{OH})\left(\mathrm{OCH}_{3}\right)$ organic intermediate and promotes the growth of NPs in specified direction leading to faceted NPs. ${ }^{6}$ Please note that capping effect of PVP also contributes a significant role in the faceting of the particles enclosed in the CTAB micelles. ${ }^{55,56}$ Fourier transform infrared spectroscopy (FTIR) data shown in Fig. 5 confirms the presence of $-\mathrm{OH}$ group at $3300 \mathrm{~cm}^{-1}$ and $-\mathrm{CH}_{3}$ group $\left(2927 \mathrm{~cm}^{-1}\right)$ in $\mathrm{PVP} / \mathrm{Mg}(\mathrm{Ac})_{2} \cdot 4 \mathrm{H}_{2} \mathrm{O} /$ $\mathrm{CTAB}$ composite fiber confirming the presence of $\mathrm{Mg}(\mathrm{OH})\left(\mathrm{OCH}_{3}\right)$ molecular intermediate which is completely absent in the $\mathrm{PVP} / \mathrm{Mg}(\mathrm{Ac})_{2} \cdot 4 \mathrm{H}_{2} \mathrm{O}$ composite fiber. The micelles become stable on stirring for 5-15 $\mathrm{h}$ and also get distributed uniformly in the solution. Furthermore, confirmation on the formation of NPs on fiber surface can be understood from Thermo Gravimetric Analysis (TGA) shown in Fig. 5 of PVP/ $\mathrm{Mg}(\mathrm{Ac})_{2} \cdot 4 \mathrm{H}_{2} \mathrm{O} / \mathrm{CTAB}$ and $\mathrm{PVP} / \mathrm{Mg}(\mathrm{Ac})_{2} \cdot 4 \mathrm{H}_{2} \mathrm{O}$ composite fibers. From both the graphs (a) \& (b), it is clear that CTAB decomposes first as its decomposition temperature is $\sim 250{ }^{\circ} \mathrm{C}$ (ref. 57 ) followed by $\mathrm{Mg}(\mathrm{Ac})_{2} \cdot 4 \mathrm{H}_{2} \mathrm{O}\left(T_{\text {decomp }} \sim 375^{\circ} \mathrm{C}\right)^{58}$ and finally PVP $\left(T_{\text {decomp }} \sim 480{ }^{\circ} \mathrm{C}\right) .{ }^{59}$ The $\mathrm{Mg}(\mathrm{OH})\left(\mathrm{OCH}_{3}\right)$ species in the micelles decompose while calcined in air to form $\mathrm{MgO}$ without losing the faceted particle morphology. Non-uniform growth and the observed morphology of the NPs on the surface can be understood due to the formation of polymer micelle complex and the combined capping effects of the polymer and surfactant. Moreover we also found that heating of electrospinning solution at $35{ }^{\circ} \mathrm{C}$ prior to electrospinning plays an important role in the observation of above-mentioned hierarchical structure of $\mathrm{NP} / \mathrm{NF}$ composite. As the non-heated electrospinning solution with CTAB in the case of ethanol, DMF + ethanol and DMF + methanol did not show the formation of such morphology. In the solution preparation we combine stirring and heating of the solution at $35{ }^{\circ} \mathrm{C}$ for 5-10 minutes before spinning. Using DMF as solvent, the stirring of the solution for long hours only lead to a turbid milky solution and a clear solution was only achieved followed by heating the solution. The experimental observation clearly shows that heating of the solution to $35^{\circ} \mathrm{C}$ is playing an

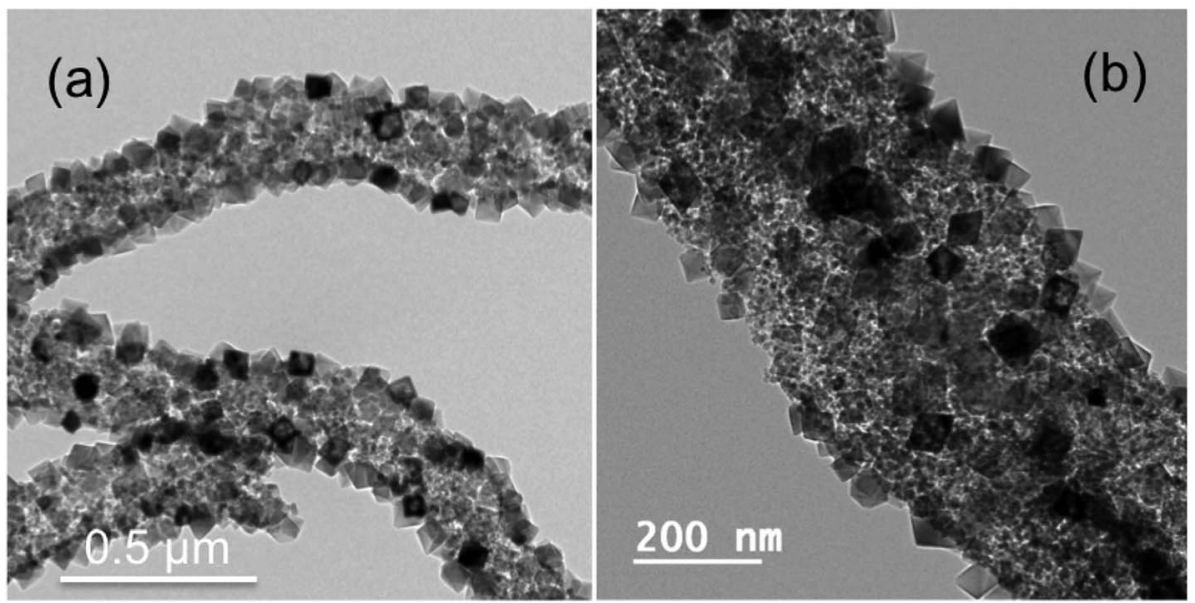

Fig. 4 (a) Bright field TEM image of MgO nanoparticle decorated MgO fibers prepared using a solvent mixture of (DMF + methanol). (b) TEM image at high magnification showing the uniform distribution of octahedron shaped MgO NPs decorated on MgO NFs. 

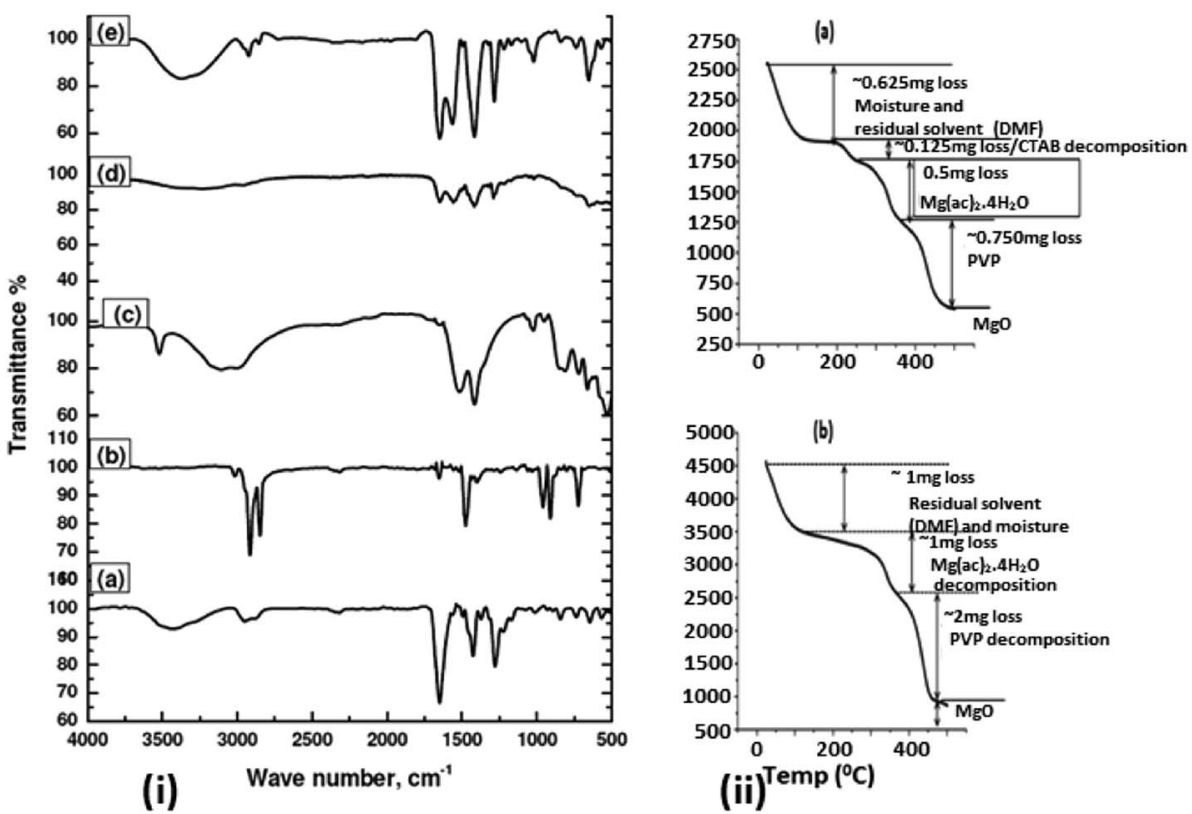

Fig. 5 (i) Fourier transform infrared spectroscopy (FTIR) of (a) PVP, (b) CTAB, (c) $M g(a c)_{2} \cdot 4 \mathrm{H}_{2} \mathrm{O}$, (d) $M g(a c)_{2} \cdot 4 \mathrm{H}_{2} \mathrm{O} / \mathrm{PVP}$ composite fiber and (e) $\mathrm{Mg}(\mathrm{ac})_{2} \cdot 4 \mathrm{H}_{2} \mathrm{O} / \mathrm{PVP} / \mathrm{CTAB}$ composite fiber. (ii) Thermogravimetric analysis of (a) $\mathrm{Mg}(\mathrm{ac})_{2} \cdot 4 \mathrm{H}_{2} \mathrm{O} / \mathrm{PVP} / \mathrm{CTAB}$ composite fiber and (b) Mg(ac) ${ }_{2}^{-}$ $\cdot 4 \mathrm{H}_{2} \mathrm{O} / \mathrm{PVP}$ fiber.

important role in the stability and formation of micelles. Since the CMC value for CTAB will depend on the added solvent mixture and the corresponding Krafft temperature where the micelle formation starts to occur. ${ }^{54}$ The increase in temperature is allowing the stabilization of micelles or the decrease in CMC value above room temperature, which enhances the micelle formation at that temperature. In addition an increased temperature enhances the micelle formation by weakening the hydration of the surfactant's hydrophilic group. ${ }^{53}$

The hollow fiber formation observed in all the cases (ethanol, methanol, DMF) can be attributed to the phase separation of PVP and $\mathrm{Mg}(\mathrm{Ac})_{2} \cdot 4 \mathrm{H}_{2} \mathrm{O}$ due to solvent evaporation. PVP has a strong interaction with the solvents than the precursor-solvents and PVP- precursor. ${ }^{60}$ During the process of electrospinning and collection of fibers, the solvent starts evaporating and PVP starts to phase separate from the metal oxide precursor and moves along the direction of increasing concentration gradient of the solvent, which is to the center of the fiber. ${ }^{61}$ Later during calcination of asspun fibers, the complete decomposition of $\mathrm{Mg}(\mathrm{Ac})_{2} \cdot 4 \mathrm{H}_{2} \mathrm{O}$ takes place first since its decomposition temperature is lesser in comparison to PVP. The decomposition of magnesium acetate produces $\mathrm{Mg}^{2+}$ ions that react with oxygen at the fiber surface to first nucleate and later to the formation of $\mathrm{MgO}$ that can be understood from Fig. 5 . Further the $\mathrm{Mg}(\mathrm{Ac})_{2} \cdot 4 \mathrm{H}_{2} \mathrm{O}$ present in the central polymer rich region also diffuses towards the surface due to the concentration gradient of $\mathrm{Mg}(\mathrm{Ac})_{2} \cdot 4 \mathrm{H}_{2} \mathrm{O}$ that exists from

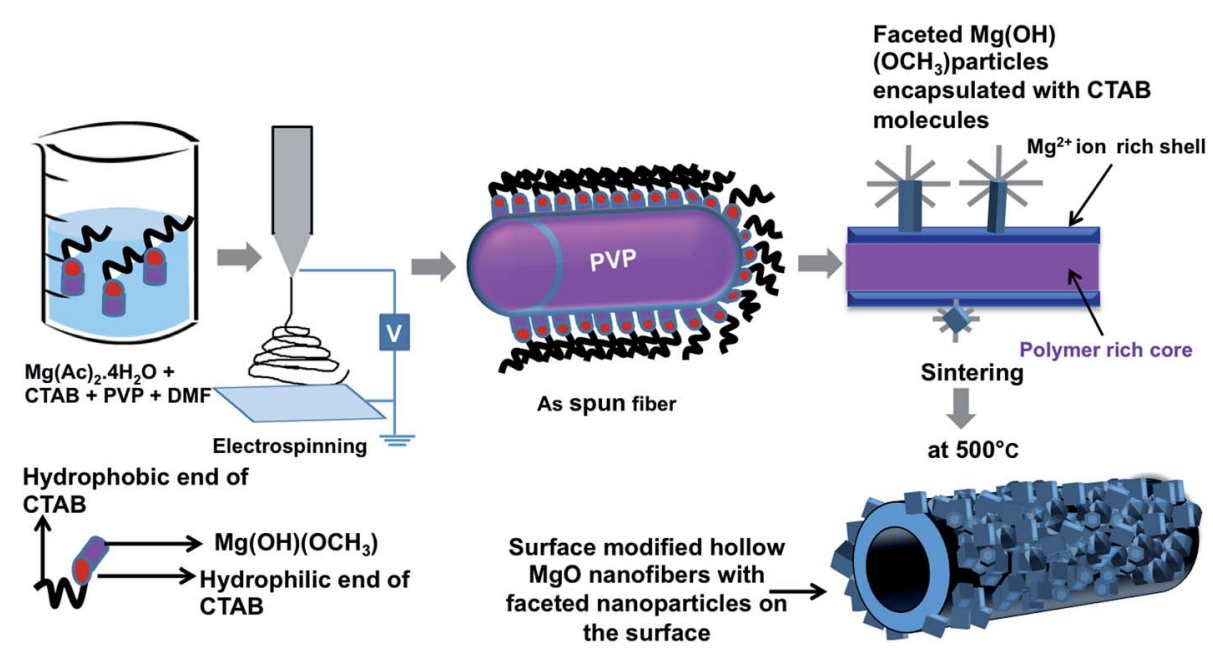

Fig. 6 Schematic diagram showing the formation of surface modified hollow fibers via electrospinning. 
inside to outside and finally the formation of $\mathrm{MgO}$ shell with PVP core occurs. On further increase in the temperature, PVP which acted as a template for hollow fiber formation, starts decomposing and the gaseous products thus formed diffuses out pulling the $\mathrm{MgO}$ particles to align together forming the shell of hollow fiber and making it porous as well. ${ }^{62}$

Moreover, the formation of faceted NPs on the fiber surface can be understood by the following explanation (Fig. 6 - schematic diagram). Initially when PVP and $\mathrm{Mg}(\mathrm{Ac})_{2} \cdot 4 \mathrm{H}_{2} \mathrm{O}$ are mixed in $\mathrm{DMF}, \mathrm{Mg}^{2+}$ ions interacts either with the oxygen atom or the nitrogen atom in the pyrrolidone ring of PVP forming a metal ion-polymer complex (Fig. 4). Once CTAB is added, it first forms a complex with polymer and later forms micelles, which encloses the molecular intermediate $\mathrm{Mg}(\mathrm{OH})\left(\mathrm{OCH}_{3}\right)$ nuclei from precursor molecules in the solution. Later, on stirring for $5-15 \mathrm{~h}$ at room temperature and followed by heating at $35{ }^{\circ} \mathrm{C}$, the formed micelles become stable and tend to uniformly disperse throughout the solution. This clear difference in the formation of NPs on the fiber surface can be due to the increased stability of the CTAB micelles at the increased temperature that holds the particles inside intact for the observed morphology. From the FTIR graph shown in Fig. 5 it is evident that the positive head group $\left(\mathrm{CTA}^{+}\right)$of $\mathrm{CTAB}$ attaches to the hydroxyl group of precursor. On electrospinning, phase separation between PVP and precursor molecules starts to occur as the solvent evaporates, leading to a core-shell morphology with polymer rich region forming the core and precursor rich region forming the shell. In this process, CTAB micelles enclosed with $\mathrm{Mg}(\mathrm{OH})\left(\mathrm{OCH}_{3}\right)$ molecular intermediate are driven to the surface of the fiber as a consequence of phase separation and also during electrospinning they are driven to the surface as they cannot be embedded in the fiber body unless the micelle size is less or concentration of CTAB is very high. Moreover, surface energy minimization and preferential adsorption of CTAB on $\{111\}$ or $\{100\}$ planes drives the NP to be faceted and form polyhedral morphologies. Furthermore during calcination in open air removes the CTAB molecules with the conversion of $\mathrm{Mg}(\mathrm{OH})\left(\mathrm{OCH}_{3}\right)$ molecular intermediate to pure $\mathrm{MgO}$. Finally this results in the formation of hierarchical hollow MgO nanofiber-nanoparticle composites.

\section{Conclusion}

We have reported the surfactant (CTAB)-driven direct synthesis of hierarchical hollow MgO NFs decorated with faceted $\mathrm{MgO}$ NPs by electrospinning. By increasing the concentration of $\mathrm{CTAB}$, faceting of nanoparticles and fiber diameter were controlled. We report for the first time that the coverage of faceted NPs and its distribution can be controlled by the order of CTAB addition to the electrospinning solution. This is mainly due to the enhanced interaction of CTAB molecules with precursor molecules. Moreover employing a mixed solvent approach of methanol $+\mathrm{DMF}$, the possibility of controlling the shape and size of the faceted NPs were feasible in comparison to pure DMF. The formation of micelles and their stability in the electrospinning is a crucial factor in determining the above hierarchical structure. Further a possible mechanism for the formation of fiber-particle morphology and hollow fiber formation has been proposed using TGA and FTIR. Finally this novel yet simple protocol for the synthesis of faceted NPs and hollow NFs composite can be easily extended/extrapolated to other material combination for various applications.

\section{Acknowledgements}

We would like to acknowledge the financial support from Science and Engineering Research Board (SERB) India through Early Career Research Award (ECR/2016/001108/ES). We also would like to acknowledge Sajin. P, Arya Raju and Dr Ramanathaswamy Pandian for electron microscopy analysis of our samples.

\section{References}

1 Y. Xia, Y. Xiong, B. Lim and S. E. Skrabalak, Angew. Chem., Int. Ed., 2009, 48, 60-103.

2 S. T. Aruna and A. S. Mukasyan, Curr. Opin. Solid State Mater. Sci., 2008, 12, 44-50.

3 D. Li, J. T. McCann, Y. Xia and M. Marquez, J. Am. Ceram. Soc., 2006, 89, 1861-1869.

4 K. Byrappa and T. Adschiri, Prog. Cryst. Growth Charact. Mater., 2007, 53, 117-166.

5 A. A. Al-Ghamdi, F. Al-Hazmi, F. Alnowaiser, R. M. AlTuwirqi, A. A. Al-Ghamdi, O. A. Alhartomy, F. El-Tantawy and F. Yakuphanoglu, J. Electroceram., 2012, 29, 198.

6 J. Pal and T. Pal, Nanoscale, 2015, 7, 14159-14190.

7 R. Narayanan and M. A. El-Sayed, J. Am. Chem. Soc., 2004, 126, 7194-7195.

8 G. Tourillon, A. Cassuto, Y. Jugnet, J. Massardier and J. Bertolini, J. Chem. Soc., Faraday Trans., 1996, 92, 48354841.

9 J. W. Hong, S.-U. Lee, Y. W. Lee and S. W. Han, J. Am. Chem. Soc., 2012, 134, 4565-4568.

10 Y. Xu, H. Wang, Y. Yu, L. Tian, W. Zhao and B. Zhang, J. Phys. Chem. C, 2011, 115, 15288-15296.

11 G. Fazio, L. Ferrighi and C. Di Valentin, J. Phys. Chem. C, 2015, 119, 20735-20746.

12 J. Yin, Z. Yu, F. Gao, J. Wang, H. Pang and Q. Lu, Angew. Chem., 2010, 122, 6472-6476.

13 L. Hu, Q. Peng and Y. Li, J. Am. Chem. Soc., 2008, 130, 1613616137.

14 X. Han, M. Jin, S. Xie, Q. Kuang, Z. Jiang, Y. Jiang, Z. Xie and L. Zheng, Angew. Chem., 2009, 121, 9344-9347.

15 Y. Jia, T. Luo, X.-Y. Yu, B. Sun, J.-H. Liu and X. J. Huang, RSC Adv., 2013, 3, 5430-5437.

16 W.-H. Ryu, H. Wilson, S. Sohn, J. Li, X. Tong, E. Shaulsky, J. Schroers, M. Elimelech and A. D. Taylor, ACS Nano, 2016, 10, 3257-3266.

17 J. Wang, G. Yang, W. Lyu and W. Yan, J. Alloys Compd., 2016, 659, 138-145.

18 L. Cheng, S. Y. Ma, T. T. Wang, X. B. Li, J. Luo, W. Q. Li, Y. Z. Mao and D. Gz, Mater. Lett., 2014, 131, 23-26. 
19 S. M. Hwang, S. Y. Kim, J.-G. Kim, K. J. Kim, J.-W. Lee, M.-S. Park, Y.-J. Kim, M. Shahabuddin, Y. Yamauchi and J. H. Kim, Nanoscale, 2015, 7, 8351-8355.

20 I.-D. Kim, E.-K. Jeon, S.-H. Choi, D.-K. Choi and H. L. Tuller, J. Electroceram., 2010, 25, 159-167.

21 R. Murugan, K. Ramamoorthy, S. Sundarrajan and S. Ramakrishna, Tetrahedron, 2012, 68, 7196-7201.

22 G. Yang, W. Yan, Q. Zhang, S. Shen and S. Ding, Nanoscale, 2013, 5, 12432-12439.

23 S. Wei, M. Zhou and W. Du, Sens. Actuators, B, 2011, 160, 753-759.

24 Z.-M. Huang, Y.-Z. Zhang, M. Kotaki and S. Ramakrishna, Compos. Sci. Technol., 2003, 63, 2223-2253.

25 W. E. Teo and S. Ramakrishna, Nanotechnology, 2006, 17, R89.

26 P. K. Panda, Trans. Indian Ceram. Soc., 2007, 66, 65-76.

27 X. Lu, C. Wang and Y. Wei, Small, 2009, 5, 2349-2370.

28 H. Xia, C. Hong, X. Shi, B. Li, G. Yuan, Q. Yao and J. Xie, J. Mater. Chem. A, 2015, 3, 1216-1221.

29 C. Wang, C. Shao, X. Zhang and Y. Liu, Inorg. Chem., 2009, 48, 7261-7268.

30 G. S. Anjusree, T. G. Deepak, S. V. Nair and A. S. Nair, J. Energy Chem., 2015, 24, 762-769.

31 Y. Li, J. Xu, T. Feng, Q. Yao, J. Xie and H. Xia, Adv. Funct. Mater., 2017, 27, 1606728.

32 H. Xia, W. Xiong, C. K. Lim, Q. Yao, Y. Wang and J. Xie, Nano Res., 2014, 7, 1797.

33 E. Formo, E. Lee, D. Campbell and Y. Xia, Nano Lett., 2008, 8, 668-672.

34 J. Wang, G. Yang, W. Lyu and W. Yan, J. Alloys Compd., 2016, 659, 138-145.

35 D. Li, J. T. McCann and Y. Xia, Small, 2005, 1, 83-86.

36 F. A. Sheikh, M. A. Kanjwal, H. Kim, D. R. Pandeya, S. T. Hong and H. Y. Kim, J. Ceram. Process. Res., 2010, 11, 685-691.

37 H. Zhang, H. Nie, D. Yu, C. Wu, Y. Zhang, C. J. B. White and L. Zhu, Desalination, 2010, 256, 141-147.

38 S. Nandan, T. G. Deepak, S. V. Nair and A. S. Nair, Dalton Trans., 2015, 44, 9637-9645.

39 S. H. Nam, H.-S. Shim, Y.-S. Kim, M. A. Dar, J. G. Kim and W. B. Kim, ACS Appl. Mater. Interfaces, 2010, 2, 2046-2052.

40 N. Sutradhar, A. B. Sinhamahapatra, S. K. Pahari, P. Pal, H. C. Bajaj, I. Mukhopadhyay and A. Panda, J. Phys. Chem. C, 2011, 115, 12308-12316.
41 Y. Y. Li, M. M. Wan, W. G. Lin, Y. Wang and J.-H. Zhu, J. Mater. Chem. A, 2014, 2, 12014-12022.

42 X.-Y. Yu, T. Luo, Y. Jia, Y.-X. Zhang, J.-H. Liu and X. J. Huang, J. Phys. Chem. C, 2011, 115, 22242-22250.

43 K. Kaviyarasu and P. A. Devarajan, Adv. Appl. Sci. Res., 2011, 2, 131-138.

44 S. Xie, Y. Wang, Q. Zhang, W. Deng and Y. Wang, ACS Catal., 2014, 4, 3644-3653.

45 K. Mageshwari and R. Sathyamoorthy, Trans. Indian Inst. Met., 2012, 65, 49-55.

46 G. Hota, S. Sundarrajan, S. Ramakrishna and N. WunJern, J. Am. Ceram. Soc., 2009, 92, 2429-2433.

47 H. Fong, I. Chun and D. Reneker, Polymer, 1999, 40, 45854592.

48 L. Jia and X.-H. Qin, J. Therm. Anal. Calorim., 2013, 112, 595605.

49 R. Nagarajan, C. Drew and C. M. Mello, J. Phys. Chem. C, 2007, 111, 16105-16108.

50 Z. M. Sui, X. Chen, L. Y. Wang, L. M. Xu, W. C. Zhuang, Y. C. Chai and C. Yang, Phys. E, 2006, 33, 308-314.

51 M. T. Anderson, J. E. Martin, J. G. Odinek and P. P. Newcomer, Chem. Mater., 1998, 10, 1490-1500.

52 M. Unger, B. Harnacke, I. Noda and H. W. Siesler, Appl. Spectrosc., 2011, 65, 892-900.

53 H.-l. Zhang, Y. Zhu, K. Zhang, T.-t. Hou, H.-y. Liu, M.-j. Cui, G.-z. Li and L. Yu, J. Solution Chem., 2009, 38, 187-198.

54 R. Nagarajan, C. Drew and C. M. Mello, J. Phys. Chem. C, 2007, 111, 16105-16108.

55 I. Pastoriza-Santos and L. M. Liz-Marzán, Adv. Funct. Mater., 2009, 19, 679-688.

56 M. S. Ali, M. Suhail, G. Ghosh, M. Kamil and others, Colloids Surf., A, 2009, 350, 51-56.

57 J. Goworek, A. Kierys, W. Gac, A. Borówka and R. Kusak, J. Therm. Anal. Calorim., 2009, 96, 375-382.

58 K. Isa and M. Nogawa, Thermochim. Acta, 1984, 75, 197-206.

59 Y. K. Du, P. Yang, Z. G. Mou, N. P. Hua and L. Jiang, J. Appl. Polym. Sci., 2006, 99, 23-26.

60 K. Nasouri, A. M. Shoushtari and M. R. M. Mojtahedi, Adv. Polym. Technol., 2015, 34, 1098-2329.

61 X. H. Li, C. L. Shao and Y. C. Liu, Langmuir, 2007, 23, 1092010923.

62 H. Xiang, Y. Long, X. Yu, X. Zhang, N. Zhao and J. Xu, CrystEngComm, 2011, 13, 4856-4860. 